\title{
LOW FIELD RELAXATIONAL BEHAVIOR OF TAAP SINGLE CRYSTAL $\left(20-10^{6} \mathrm{~Hz}\right)$
}

\author{
J.R. Fernández del Castillo ${ }^{a}$, T. Iglesias $^{a}$, J. Przeslawski ${ }^{b}$ \\ AND J.A. GONZALO ${ }^{a}$ \\ ${ }^{a}$ Departamento de Física de Materiales, C-IV, Universidad Autónoma de Madrid \\ 28049 Madrid, Spain \\ ${ }^{b}$ Institute of Experimental Physics, University of Wrocław \\ Pl. Maxa Borna 9, 50-205 Wrocław, Poland
}

(Received October 3, 1997; in final form December 8, 1997)

\begin{abstract}
Relaxational behavior of ferroelectric telluric acid ammonium phosphate crystals were investigated in the range $20 \mathrm{~Hz}$ to $1 \mathrm{MHz}$ by means of low field measurements of $\varepsilon^{\prime}$ and $\tan \delta$ in the vicinity of ferroelectric phase transitions at a number of frequencies. Results are compared with those for triglycine selenate, a much "softer" ferroelectric material. The measuring field and frequency dependence of the effective potential barrier height for domain wall motion are compared in both crystals.
\end{abstract}

PACS numbers: $64.60 . \mathrm{Fr}, 77.80 .-\mathrm{e}$

\section{Introduction}

Telluric acid ammonium phosphate (TAAP), of chemical formula $\left[\mathrm{Te}(\mathrm{OH})_{6}\right] \cdot 2\left[\mathrm{NH}_{4} \mathrm{H}_{2} \mathrm{PO}_{4}\right] \cdot\left[\left(\mathrm{NH}_{4}\right)_{2} \mathrm{HPO}_{4}\right]$, is a relatively new ferroelectric crystal [1]. TAAP is known to be a rather "hard" ferroelectric (high coercive field) in comparison with other hydrogen-bonded materials, e.g. members of the triglycine sulfate (TGS) family. Relaxational behavior of TAAP was previously studied at a frequency range $10 \mathrm{kHz}-0.4 \mathrm{MHz}$ and in a microwave band $1 \mathrm{MHz}-77 \mathrm{GHz}[2,3]$.

A single relaxational soft mode frequency follows the Curie-Weiss law, which confirms the order-disorder character of the ferroelectric transition in TAAP [3].

We measured the dielectric permittivity $\varepsilon^{\prime}$ and the loss factor $D=\tan \delta=$ $\varepsilon^{\prime \prime} / \varepsilon^{\prime}$ in the vicinity of $T_{\mathrm{c}}$ in the frequency range $20 \mathrm{~Hz}-1 \mathrm{MHz}$. Data were analyzed and compared with similar data taken for triglycine selenate (TGSe) crystal. 


\section{Experimental}

TAAP and TGSe samples were cut perpendicularly to the spontaneous polarization direction from crystals of very good quality. Gold leaf electrodes were attached to the main surfaces of the samples. Dielectric permittivity and loss factor data were collected by means of a HP Precision LCR Meter 4384A, connected to a computer, that also recorded the temperature via readings of the thermoelectric voltage from a copper-constantan thermocouple with a Keithley 196 DMM digital multimeter. The measuring field amplitude varied from 10 to $50 \mathrm{~V} / \mathrm{cm}$ for the TAAP crystal and from 0.5 to $7 \mathrm{~V} / \mathrm{cm}$ for the TGSe crystal.

\section{Results and discussion}

A relaxation-like frequency was obtained from the Debye relationship [4]: $f=1 / \tau \cong \omega / D$, where $\omega$ is the measuring frequency. Figure 1 gives these frequencies vs. $1 / T$, obtained for constant measuring field, at various frequencies and amplitudes for both crystals (TAAP and TGSe). Note the logarithmic scale and the different low frequency dependence $f(\omega)$ for TAAP and TGSe crystals. There is a gradual change of $f(1 / T)$ behavior with frequency for TAAP (Fig. 1a) as well as for TGSe (Fig. 1b).
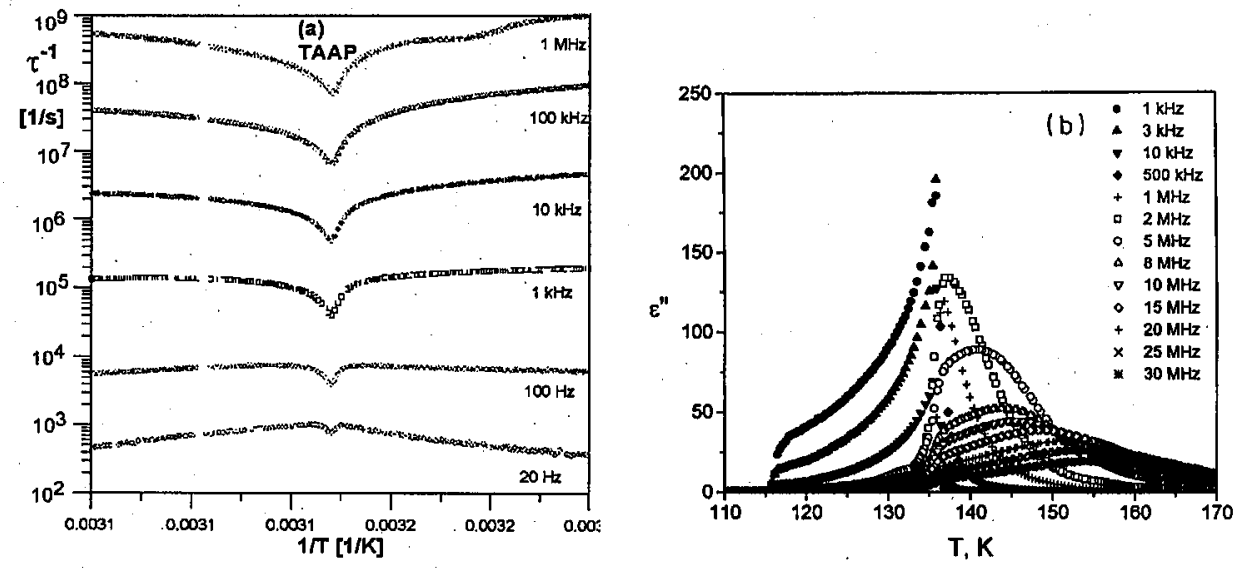

Fig. 1. Inverse domain wall relaxation time $\left(\tau^{-1}\right)$ vs. $1 / T$ for TAAP (a) and for TGSe (b) at various frequencies.

In Fig. 2 shapes of the reciprocal temperature dependence of the relaxational frequency $f_{\mathrm{w}}(T)=\tau^{-1}(T)$ are presented at the chosen measuring field frequency of $1 \mathrm{kHz}$ for both crystals, showing similar behavior for very different field amplitudes ( $E_{0}=10 \mathrm{~V} / \mathrm{cm}$ for TAAP, $E_{0}=0.5 \mathrm{~V} / \mathrm{cm}$ for TGSe). Below the ferroelectric transition a local maximum and then a decline of the relaxational frequency is observed. The phase transition temperature is characterized by a pronounced minimum of the relaxational frequency. Similar temperature variation of the relaxational frequency estimated from maxima of $\varepsilon^{\prime \prime}(\omega)$ at constant temperatures was found for TGSe single crystals $[5,6]$. The steep decrease in $\tau^{-1}(T)$ at $T$ close to 

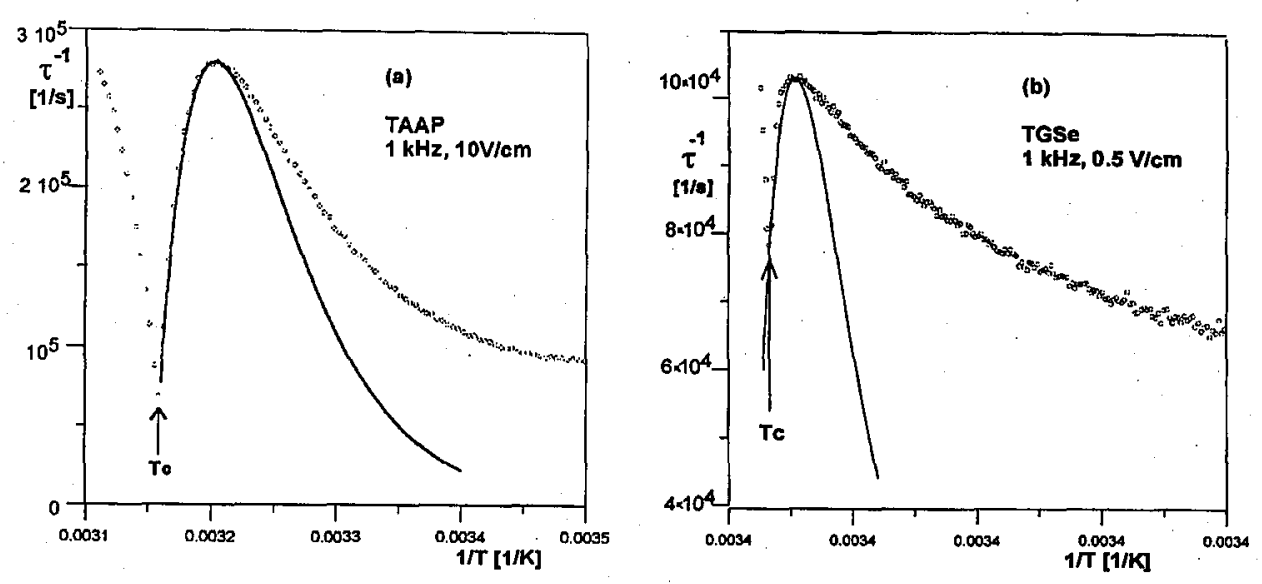

Fig. 2. Fit of $\tau^{-1}(1 / T)$ to Eq. (4) for TAAP (a) with $E=10 \mathrm{~V} / \mathrm{cm}$, TGSe (b) with $E=0.5 \mathrm{~V} / \mathrm{cm}$ at $1 \mathrm{kHz}$.

$T_{\mathrm{c}}$ is similar to the behavior at the intrinsic relaxation frequency $\left(\approx 10^{9} \mathrm{~Hz}\right)$. The decrease in $f$ which takes place further away from $T_{c}$ can be connected with the influence of the Boltzmann factor appearing in $\tau^{-1}(T)$, with a potential barrier much larger than that for individual dipoles. This is related to the slowing down of the domain wall motion.

The inverse intrinsic (single dipole) relaxation time for a ferroelectric can be written as

$$
\frac{1}{\tau_{\mathrm{r}}}=2 \frac{\Delta T}{T}\left(\frac{k_{\mathrm{B}}}{h}\right) \mathrm{e}^{-\Delta U / k_{\mathrm{B}} T} \quad\left(T \leq T_{\mathrm{c}}\right)
$$

Similarly, the inverse domain wall relaxation time for a ferroelectric (occurring at much lower frequencies) can be written as

$$
\frac{1}{\tau_{\mathrm{w}}}=2 \frac{\Delta T}{T}\left(\frac{k_{\mathrm{B}}}{h}\right) \sum_{n=1}^{\infty} \mathrm{e}^{-n \Delta U_{\mathrm{w}} / k_{\mathrm{B}} T}
$$

where the probability for successive jumps $(n=1,2, \ldots)$ is considered, and $\Delta U_{\mathrm{w}}$ is the energy barrier for domain wall motion.

The experimental data for $1 / \tau_{\mathrm{w}}$ vs. $1 / T$ go through a maximum at $\left(T_{\mathrm{c}}-\Delta T\right)$ about $5^{\circ} \mathrm{C}$ to $10^{\circ} \mathrm{C}$. From Eq. (2) the maximum can be determined making $\mathrm{d}\left(1 / \tau_{\mathrm{w}}\right) / \mathrm{d}(1 / T)=0$, which implies

$$
\frac{T_{\mathrm{m}}}{T_{\mathrm{c}}} \cong \frac{\Delta U_{\mathrm{w}} / k_{\mathrm{B}} T_{\mathrm{m}}}{1+\left(\Delta U_{\mathrm{w}} / k_{\mathrm{B}} T_{\mathrm{m}}\right)}
$$

where $T_{\mathrm{m}}$ is the temperature of the maximum of $1 / \tau_{\mathrm{w}}$ versus the inverse of temperature in the ferroelectric phase.

Then

$$
\begin{aligned}
& \frac{f_{\mathrm{w}}(T)}{f_{\mathrm{w}}\left(T_{\mathrm{m}}\right)}=\frac{\left[\left(1-\exp \left(-\Delta U_{\mathrm{w}} / k_{\mathrm{B}} T\right)\right)^{-1}-1\right] \Delta T}{\left[\left(1-\exp \left(-\Delta U_{\mathrm{w}} / k_{\mathrm{B}} T_{\mathrm{m}}\right)\right)^{-1}-1\right] \Delta T_{\mathrm{m}}} \\
& \quad \approx \frac{\Delta T}{\Delta T_{\mathrm{m}}} \exp \left[-T_{\mathrm{w}}\left(\frac{1}{T}-\frac{1}{T_{\mathrm{m}}}\right)\right] .
\end{aligned}
$$



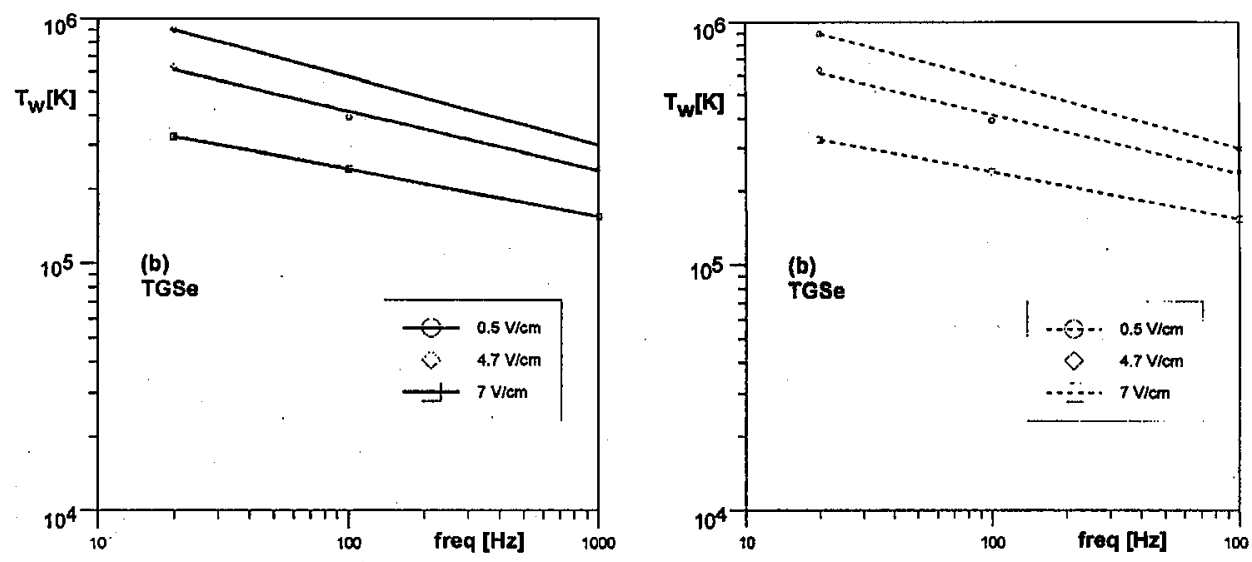

Fig. 3. Effective equivalent temperature of barrier height $T_{\mathrm{w}}=\Delta U_{\mathrm{w}} / k_{\mathrm{B}}$ for domain wall motion for TAAP $\left(10^{2}\right.$ to $\left.10^{4}\right)(\mathrm{a})$ and for TGSe $\left(10\right.$ to $\left.10^{3}\right)(\mathrm{b})$ at various fields.

We define $\Delta T_{\mathrm{m}}$ as the difference between $T_{\mathrm{c}}$ and $T_{\mathrm{m}}$. The fits for TAAP and TGSe are shown in Fig. 2a, b.

We estimated $T_{\mathrm{w}}=\Delta U_{\mathrm{w}} / k_{\mathrm{B}}$ values for various measuring frequencies and field amplitudes. The frequency dependence of the $T_{\mathrm{w}}$ is shown in Fig. 3a, b. For TAAP it seems to go through a barely observed minimum of $T_{\mathrm{w}}$ at about $10 \mathrm{kHz}$ for the lowest field amplitude $(10 \mathrm{~V} / \mathrm{cm})$. In the case of TGSe the data could be analyzed only up to a measuring frequency of $1 \mathrm{kHz}$. For higher frequencies the maximum of the relaxational frequency below $T_{c}$ was not within our experimental window. Significant differences in the field and amplitude dependence of the $T_{\mathrm{w}}$ may be expected for both crystals outside the field and frequency ranges shown. A tendency to a fixed $T_{\mathrm{w}}$ value at higher fields was observed for TAAP crystals, whereas $T_{\mathrm{w}}$ was still somewhat field and frequency dependent for TGSe crystals. -It may be said that in order to get similar behavior a higher measuring field was needed for TAAP than for TGSe crystals.

The behavior with temperature of domain wall relaxation frequency at $T_{\mathrm{c}} \leq \Delta T$ is therefore semiquantitatively described by Eq. (4) for TAAP ("hard ferroelectric") and for TGSe ("soft ferroelectric").

\section{Acknowledgments}

We acknowledge financial support from CICyT, grant No. PB93-1253; from Iberdrola, grant INDES 94/95, and to the Comunidad de Madrid, project No. AE00138/94.

\section{References}

[1] S. Guillot Gauthier, J.C. Peuzin, M. Olivier, G. Rolland, Ferroelectrics 52, 293 (1984).

[2] M.N. Shashikala, M.R. Srinivasan, H.L. Bhat, J. Phys., Condens. Matter 2, 4013 (1990). 
[3] R. Sobiestianskas, J. Grigas, Z. Czapla, Phase Transit. 37, 157 (1992).

[4] P. Debye, Polar Molecules, Dover, New York 1945.

[5] J. Przesławski, T. Iglesias, J.A. Gonzalo, Solid State Commun. 96, 195 (1995).

[6] T. Iglesias, J. Przesławski, J.A. Gonzalo, Ferroelectrics 185, 313 (1996). 